\title{
Identifying the Spatiotemporal Patterns of Traditional Villages in China: A Multiscale Perspective
}

\author{
Chao Wu ${ }^{1,2}$, Miaomiao Chen ${ }^{1}$, Lei Zhou ${ }^{1,2}$, Xiaojin Liang ${ }^{3}$ and Wei Wang ${ }^{1,2, *}$ \\ 1 School of Geographic and Biologic Information, Nanjing University of Posts and Telecommunications, \\ Nanjing 210023, China; chaowu@njupt.edu.cn (C.W.); B18080605@njupt.edu.cn (M.C.); \\ zhoulei@njupt.edu.cn (L.Z.) \\ 2 Smart Health Big Data Analysis and Location Services Engineering Lab of Jiangsu Province, \\ Nanjing University of Posts and Telecommunications, Nanjing 210023, China \\ 3 School of Resources and Environmental Science, Wuhan University, Wuhan 430079, China; \\ liangxj@whu.edu.cn \\ * Correspondence: wangwei89@njupt.edu.cn; Tel.: +86-157-2704-5196
}

Received: 13 September 2020; Accepted: 10 November 2020; Published: 16 November 2020

check for updates

\begin{abstract}
Traditional villages are important carriers of traditional cultural heritage, and they have strong historical, cultural, aesthetic and tourism value for all countries and the international community. In China, the number of traditional villages is currently decreasing each year, and the precious material and non-material heritage is at risk of disappearing in the process of urbanization. A comprehensive understanding of the spatiotemporal patterns of traditional villages on multiple scales has important significance in protecting traditional culture, revitalizing traditional villages and achieving sustainable urbanization. Therefore, the spatiotemporal characteristics of traditional villages at the city, province, and geographic zone scales are explored by a series of Geographic Information System(GIS)-based methods in this article. Specifically, the analysis units are multi-scale, the applied methods are multi-variate, and the identified patterns are multi-perspective. The results demonstrate that the distribution of traditional villages in China is unbalanced over space and time. Moreover, the different spatiotemporal distributions of traditional villages are sensitive to scales. These findings clarify differences in the corresponding geographic and environmental factors, the level of economic development and local policy support. We further suggest that exploring the effective and suitable modes of protection and rural development is necessary. The results of this article revealing the unbalanced spatiotemporal distribution of traditional villages can provide valuable suggestions and insights into alleviating regional inequality in China.
\end{abstract}

Keywords: traditional village; rural geography; spatial distribution; temporal changes; multiscale; China

\section{Introduction}

Traditional villages, also called ancient villages, are non-renewable resources bearing traditional cultural heritage, and they have strong historical, cultural, scientific, social, aesthetic and economic and tourism value for all countries and the international community [1,2]. Many scholars are calling for the protection of traditional villages. They are important carriers and manifestations of agricultural and ecological civilization, and they have essential effects on the sustainability of the human settlement spatial environment, stability of the society and future growth of the entire country [3,4]. In 1989, the United Nations Educational, Scientific and Cultural Organization (UNESCO) emphasized the importance of the protection of villages for traditional culture and folk customs [5]. In China, traditional villages were first called ancient villages, and this term refers to the villages built before 1949 [6]. Approximately 
500-600 million people still live in the rural areas [7], and traditional villages can effectively reflect the relationships between people and the land and between the historical background and social politics, which have significant effects on the future growth of China [3,8-10]. Learning about the spatial and temporal distributions of these villages is of great significance to reconstructing a sustainable rural social economy and reducing the regional differences in rural transformation development [11,12].

However, with the rapid development of urbanization, many traditional villages are facing gradual decay and decline due to various artificial or natural causes in China [13-15]. While urbanization brings about the development of the rural economy, it causes the problem of low economic efficiency in rural areas [15]. A strong mix of agricultural and industrial land and poor infrastructure in rural areas leads to pollution of farmland, which in turn leads to ecological degradation of traditional villages and harms food safety and health [16]. Under the above circumstances, the Chinese government has begun to attach great importance to the issues relating to traditional villages including protection and development [17]. In December 2012, the Ministry of Housing and Urban-Rural Development of the People's Republic of China, the Ministry of Culture of the People's Republic of China and the Ministry of Finance of the People's Republic of China issued a notice to publish a list of traditional Chinese villages and the selection criteria for defining traditional villages. Until 2019, China had published five batches of traditional villages with a total number of 6819 , illustrating that a large number of valuable traditional villages have been protected and developed with great attention and promotion by the government of China (http://www.mohurd.gov.cn/wjfb/201906/t20190620240922.html).

Recently, an increasing number of scholars have explored the topic of traditional villages in China. Compared with early foreign research on traditional villages about the culture, landscape pattern, spatial form and sustainable development of traditional villages [18-22], the research on traditional Chinese villages focuses more on the revitalization, development and construction of traditional villages, including the economy, tourism, traffic accessibility and resource management $[1,6,17,23-26]$. This focus is inseparable from the fact that China is a large agricultural country, and the issues of agriculture, rural areas and farmers (i.e., the "three rural" issues) have always been major national issues [27]. Moreover, the 19th National Congress of the Communist Party of China (CPC) put forward the "strategy for rural revitalization", which provides a rare opportunity for traditional villages to achieve moderate, reasonable and sustainable development [28]. Furthermore, some scholars have noted many differences between the mature system and complete industrial chain of traditional villages in European countries and China [29]. The above studies and policies are of great significance in guiding the formulation of strategies and policies related to the reconstruction of the rural economy and the development of traditional villages, thus providing useful references for the effective promotion of urban-rural integration in China. However, the status of the Chinese countryside within the whole regional economic pattern has not been guaranteed [30]. Relevant studies on the current situation of rural areas in China and the mechanisms of urban-rural differences still need to be further strengthened and enriched, which is important for urban-rural integration and sustainable economic and social development [11,31].

A traditional village is a region interwoven with economic and social activities and has obvious comprehensive and regional characteristics. Therefore, from the perspective of geography, exploring the spatial pattern of traditional villages and their evolution characteristics is necessary and meaningful [32-34]. However, systematic studies on traditional villages from the perspective of geography, especially spatiotemporal perspectives, are still insufficient. Although some spatial research on traditional villages exists, these studies have mostly been conducted across provinces, cities or a particular village in China. The existing studies mainly focus on the temporal trend and spatial pattern at single-scale differences and pay less attention to scale sensitivity and dependence [35-38]. The spatiotemporal differentiation results of different responses of administrative divisions at different levels to regulatory policies and declaration conditions in traditional villages are the comprehensive results of top-down national strategy and bottom-up local response. In fact, fully grasping the scale differences and internal relations of the spatial and temporal distribution of traditional villages is difficult as the spatiotemporal differentiation 
and correlation of the evolution of traditional villages at different scales is ignored. In recent years, an increasing number of scholars have emphasized that the development trend and underlying mechanism of geographic phenomena at different spatial scales may vary [39-42]. That is, exploring geographic phenomena and processes at multiple scales is significant [43,44]. Because China comprises a vast space with discrepancies and inequalities in geography and the economy, the spatiotemporal dynamics and distribution of traditional villages are uneven in China. Therefore, analyzing and comparing the spatiotemporal patterns of traditional villages at different scales may be meaningful. A deep understanding of the spatiotemporal distribution characteristics and evolution rules of traditional Chinese villages is necessary to reflect the dynamics of economic inequality. Thus, this study can help formulate region-based policies to promote the development and protection of traditional villages and enhance China's competitiveness and further growth [45].

In view of this, we used multivariate GIS-based methods to explore the spatiotemporal distribution and dynamics of traditional villages on multiple scales in China and reveal multi-perspective patterns

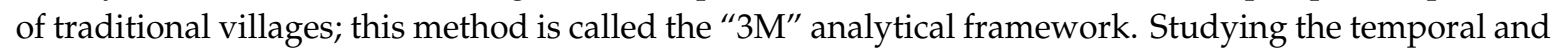
spatial distribution characteristics of different batches can reflect the evolution law of human village culture under the complex topographic elements in China, as well as the differences in the intensity and effect of the development and protection of traditional villages in different regions. We attempted to explore the inequality and dynamics of rural areas. Then, the degree of aggregation of traditional villages at different spatial scales was analyzed, and the spatial distribution of hot spots and cold spots can be further measured. Finally, a comparative multiscale distribution analysis of Chinese traditional villages was conducted based on spatial pattern identification methods. In this paper, the dynamic development and spatial pattern of Chinese traditional villages were measured and compared from the three dimensions of zone, province and prefecture. By exploring the scale effect and space effect of multi-scale villages, we hope to compensate for the deficiency of existing research results. This article provides insights into rural revitalization and coordinated rural development throughout China. Furthermore, the proposed framework can be extended to other countries and international communities to reveal the spatiotemporal patterns of villages and provide decision support for the spatial reconstruction of regional rural settlements.

\section{Study Area}

According to the standard of traditional village evaluation and identification index system (trial), the Ministry of Housing and Urban-Rural Development, Ministry of Culture and Ministry of Finance have released five batches of traditional villages, with a total number of 6819 (first batch of 646, second batch of 915, third batch of 994, fourth batch of 1598 and fifth batch of 2666). In fact, all the five batches are part of one single catalogue of similar traditional villages according uniform criterion, with one first list (batch 1) progressively enlarged with more villages (batches 2 to 5 ). A large number of traditional Chinese villages cover all of the provinces except Hong Kong, Macao and Taiwan, forming the world's largest and most valuable active agricultural civilization settlements. Notably, according to the uniform criterion of declaring traditional villages, local provincial or municipal governments conducted the preliminary evaluation and recommendation of the villages that conform to the criteria. Then, afterwards, the expert committee for the protection and development of traditional villages evaluated and identified and publicized them. The number of valuable traditional villages defined in the five batches increased year by year, and the number of traditional villages defined in the fifth batch reached a peak, accounting for $39.1 \%$ of the total number of traditional villages in China. The related materials about national traditional villages mainly came from the official website of the Ministry of Housing and Urban-Rural Development of the People's Republic of China Construction (www.mohurd.gov.cn) and the website of Chinese traditional villages (www.chuantongcunluo.com). The research period of this article ends in 2019, and more than $80 \%$ of cities in China have declared the existence of traditional villages within their area of influence according to the related criteria. Additionally, in the study of geography, an increasing number of scholars have proposed that the 
development trends and underlying mechanisms of geographic patterns and processes are sensitive to the varying spatial scales $[39,46,47]$. The distribution differences of traditional villages in China were measured and compared at three spatial scales: geographic zone and provincial and prefectural level. The research scope of this article does not include Hong Kong, Macao and Taiwan (Figure 1).

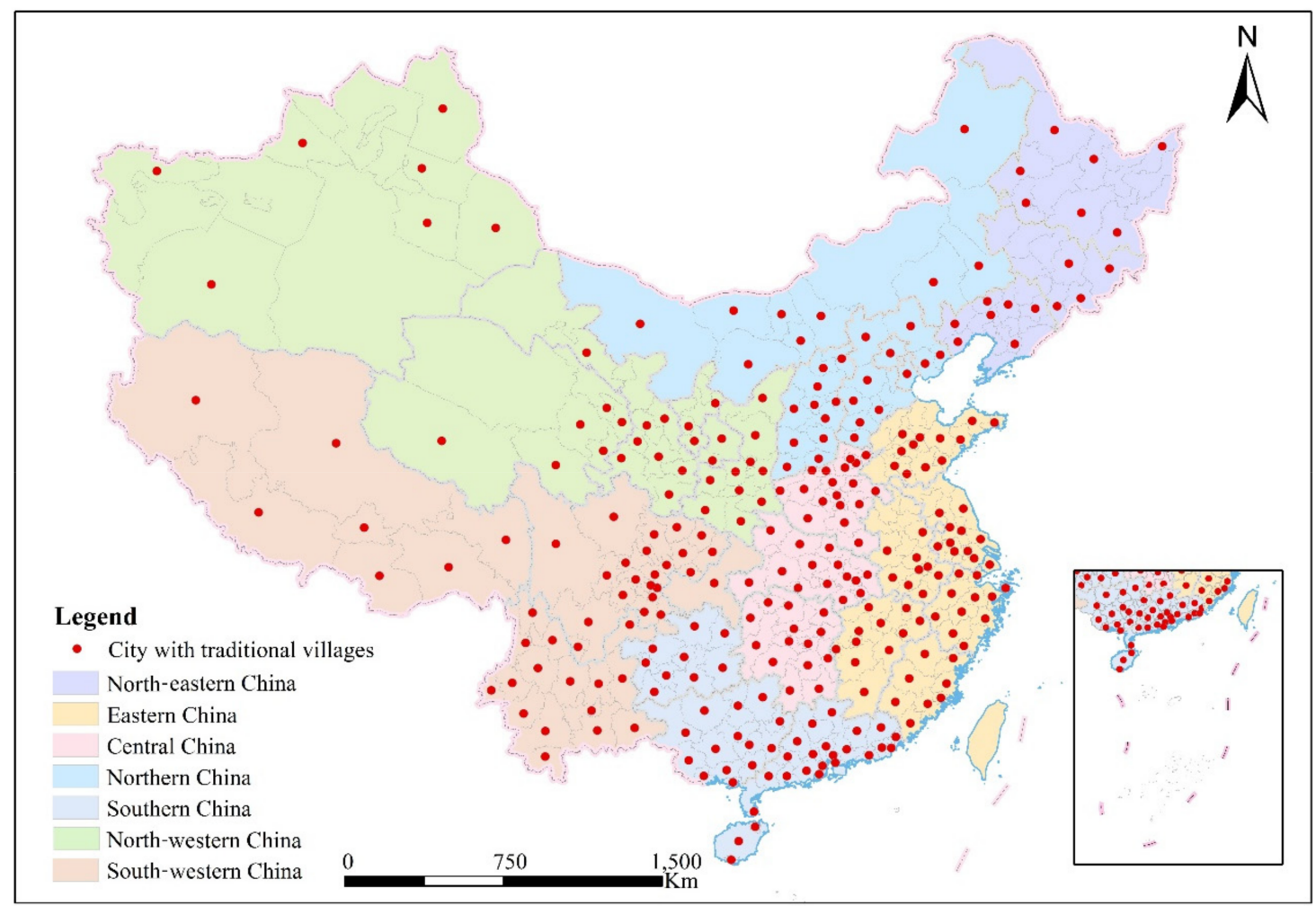

Figure 1. The study area and the distribution of cities with traditional villages.

\section{Methodology}

\section{1. "3M" Analytical Framework}

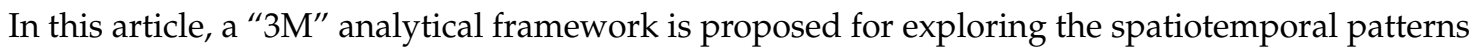
of traditional villages, and this framework includes multiscale research units and applies multivariate analytical methods to identify the multiple perspectives of the spatiotemporal patterns (Figure 2). Specifically, to measure and compare the changing trend and spatial pattern of traditional villages, this article uses regional-, provincial- and prefecture-level units to study the temporal distribution differences and their spatial patterns. The adopted multivariate methods include the spatial Gini coefficient, hot spot analysis (Getis-Ord $G_{i}^{*}$ ) and weighted standard deviation ellipse, which can reveal multiple perspectives of patterns, including degree of aggregation, regional inequality, clustering distribution, directional distribution and degree of dispersion. The three selected methods are widely used for identifying the spatial patterns in previous studies for exploring the spatial and temporal distribution patterns of geographical phenomena [48-51]. The three methods are independent and not redundant, and they can reflect the comprehensive and multilevel characteristics of the distribution and dynamics of Chinese traditional villages. The proposed " $3 \mathrm{M}$ " analytical framework in this article can explore the heterogeneity of multiscale traditional villages and reveal the scale effects and spatial effect of the uneven distribution of traditional villages throughout China. In this article, software including Python, ArcGIS and Geoda were used to calculate the above indicators and visualize the analytical results. 


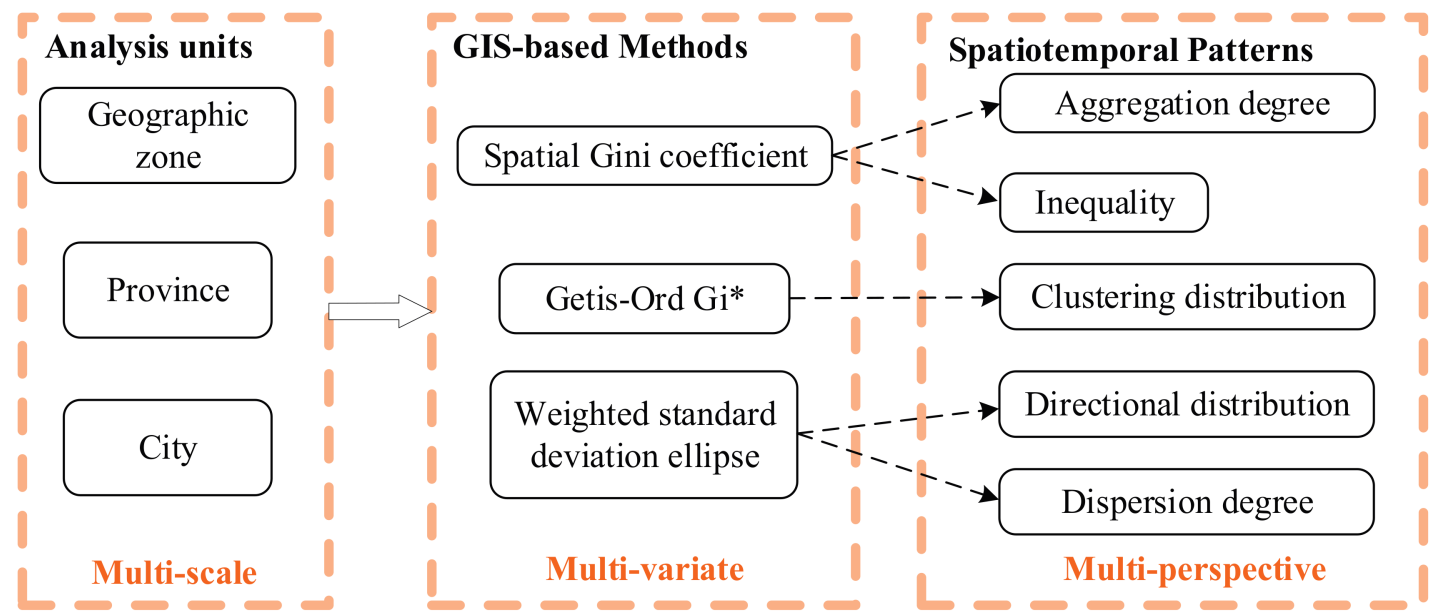

Figure 2. The " $3 \mathrm{M}$ " framework for spatiotemporal analysis of traditional villages in China.

\subsection{Spatial Gini Coefficient for Measuring Spatial Inequality}

The spatial Gini coefficient and Theil index are two complementary methods. Specifically, the spatial Gini coefficient is an important method for studying the spatial distribution of discrete regions in geography, and it is mainly used to compare the spatial distribution differences of regional geographic features [52-54]. The Gini coefficient can be applied to measure the degree of aggregation of traditional villages. The values of the Gini coefficients are within the interval $[0,1]$. If the value of the Gini coefficient is larger, the spatial distribution of traditional villages is much more unbalanced, that is, the stronger the spatial concentration is. In other words, the closer the spatial Gini coefficient is to 0 , the more even the distribution of traditional villages will be. On the contrary, the closer the spatial Gini coefficient is to 1 , the more concentrated the distribution of traditional villages will be. The Gini coefficient is based on the average deviation method and can be calculated as follows [55]:

$$
G=\frac{1}{2 n^{2} \mu_{x}} \sum_{i=1}^{n} \sum_{j=1}^{n}\left|x_{i}-x_{j}\right|
$$

where $n$ is the total number of samples. $\mu_{x}$ represents the mean value of a unit attribute at a specified scale. $x_{i}$ and $x_{j}$ are unit attribute values in the $i$ th and $j$ th unit, respectively. For example, in the province scale, $x_{i}$ and $x_{j}$ represent the number of traditional villages in the $i$ th and $j$ th province, respectively.

\subsection{Getis-Ord $G_{i}^{*}$ for Identifying Spatial Clustering Patterns}

The Getis-Ord $G_{i}^{*}$ statistic is a famous hot spot analysis method, and it has been widely used in many fields [56]; this method can be used to efficiently recognize the grouping of spatial patterns [57,58]. Through the results including the $z$ score and $p$ value, the samples of the high value or low value in the spatial clustering can be identified. $G_{i}^{*}$ is given by

$$
\left\{\begin{array}{c}
G_{i}^{*}=\frac{\sum_{j=1}^{n} w_{i j} x_{j}-\bar{X} \sum_{j=1}^{n} w_{i j}}{S \sqrt{\left[n \sum_{j=1}^{n} w_{i j}^{2}-\left(\sum_{j=1}^{n} w_{i j}\right)^{2}\right] / n-1}} \\
\bar{X}=\frac{\sum_{j=1}^{n} x_{j}}{n} \\
S=\sqrt{\sum_{j=1}^{n} x_{j}^{2} / n-(\bar{X})^{2}}
\end{array}\right.
$$

where $n$ is the total number of analysis units. $x_{j}$ represents the number of traditional villages of unit (i.e., province/city) $j$. $w_{i j}$ denotes the spatial weight between region $i$ and region $j$. In this article, 
the spatial weight is calculated based on the fixed distance band method. The $G_{i}^{*}$ statistic returned for each unit of study is the z-score. If the value of $G_{i}^{*}$ is significantly positive, then the value around region $i$ is relatively high and belongs to a hot spot [59]. Otherwise, it is a cold area.

\subsection{Weighted Standard Deviation Ellipse for Dispersion Patterns}

The standard deviation ellipse (SDE) is usually applied to describe the spatial characteristics of geographic entities, including the central tendency, dispersion and directivity $[60,61]$. The spatiotemporal distribution pattern of geographic events has obvious characteristics of spatial anisotropy. The spatial distribution of many geographic phenomena has obvious differences in the degree of dispersion in different directions. For example, the occurrence of traffic accidents is usually distributed along the road network, showing distribution characteristics similar to the morphology of main roads. Due to the spatial correlation of the regional population and economic and social development level, the spatial and temporal distribution of traditional villages studied in this paper also has the characteristics of spatial anisotropy. In this paper, the SDE method is used to conduct an in-depth analysis of the directional characteristics of traditional villages. The SDE is determined by the standard deviation (SD) of the long axis, the SD of the short axis and the rotation azimuth. The long axis and short axis represent the direction and range, respectively, of traditional village distributions. The ratio of the long axis to the short axis indicates the flatness of the SDE. The SDs of the long and short axes are calculated as follows:

$$
\left\{\begin{aligned}
S D E_{x} & =\sqrt{\sum_{i=1}^{n}\left(x_{i}-\bar{x}\right)^{2} / n} \\
S D E_{y} & =\sqrt{\sum_{i=1}^{n}\left(y_{i}-\bar{y}\right)^{2} / n}
\end{aligned}\right.
$$

where $\left(x_{i}, y_{i}\right)$ are the spatial coordinates of the $i$ th point element. $(\bar{x}, \bar{y})$ are the geometric central coordinates of the point feature class. The rotation angle is calculated as follows:

$$
\left\{\begin{array}{c}
\tan \theta=\frac{\mathrm{A}+\mathrm{B}}{\mathrm{C}} \\
A=\left(\sum_{i=1}^{n} \widetilde{x}_{i}^{2}-\sum_{i=1}^{n} \widetilde{y}_{i}^{2}\right) \\
B=\sqrt{\left(\sum_{i=1}^{n} \widetilde{x}_{i}^{2}-\sum_{i=1}^{n} \widetilde{y}_{i}^{2}\right)^{2}+4\left(\sum_{i=1}^{n} \widetilde{x}_{i} \widetilde{y}_{i}\right)^{2}} \\
C=2 \sum_{i=1}^{n} \widetilde{x}_{i} \widetilde{y}_{i}
\end{array}\right.
$$

where $\left(\widetilde{x}_{i}, \widetilde{y}_{i}\right)$ represents the deviation between the geometric center coordinates of the feature class and the coordinates of $\left(x_{i}, y_{i}\right)$. The SDs of the $x$-axis and $y$-axis are calculated as follows:

$$
\left\{\begin{array}{l}
\sigma_{x}=\sqrt{2} \sqrt{\sum_{i=1}^{n}\left(\widetilde{x}_{i} \cos \theta-\widetilde{y}_{i} \sin \theta\right)^{2} / n} \\
\sigma_{y}=\sqrt{2} \sqrt{\sum_{i=1}^{n}\left(\widetilde{x}_{i} \sin \theta+\widetilde{y}_{i} \cos \theta\right)^{2} / n}
\end{array}\right.
$$

To correct the deviation caused by different probabilities, this article chose the total number of traditional villages under the given scale as the weights for Equations (3)-(5), namely, the weighted standard deviation ellipse (WSDE) [62]. The long and short axes ( $x$-axis and $y$-axis) of the WSDE are used to represent the spatial range of the traditional village distribution, and the long axis is used as the main direction of the trend. The rotation of the elliptical azimuth can reflect the directional extension of traditional village centers. The standard deviation of the long and short axes of the ellipse can reflect the distribution and development trend and strength of traditional villages. For example, scholars can 
make a preliminary judgment whether the traditional villages in a certain region are distributed along the river or road network. If the elliptic direction of a certain region is basically consistent with the direction of the river, then the river network in this region has a great impact on the development of traditional villages.

\section{Spatiotemporal Patterns of Traditional Villages}

\subsection{Spatial Inequality of Traditional Villages}

According to Figure 3a, the spatial Gini coefficients of different research levels (i.e., zone-province-city) throughout China show obvious and significantly different degrees of polarization distribution. The temporal tendencies of the Gini coefficients of the three levels are similar. The values of the Gini coefficients in the five batches of traditional villages are, in decreasing order, as follows: city-level > province-level> geographic zone level, illustrating that the smaller the spatial scale is, the greater the spatial imbalance is in the distribution of traditional villages. This shows that the difference among prefecture-level cities is the leading factor of the total inequality. In the temporal dimension, three scales show a similar tendency of the degree of spatial concentration. According to the statistics of cities with more traditional villages, $29 \%$ of the traditional villages are clustered in the top $2.7 \%$ of prefecture-level cities, which illustrates that most of the traditional villages are concentrated in some specific provinces and counties, such as Huangshan city in Anhui province and Jincheng city in Shanxi province.
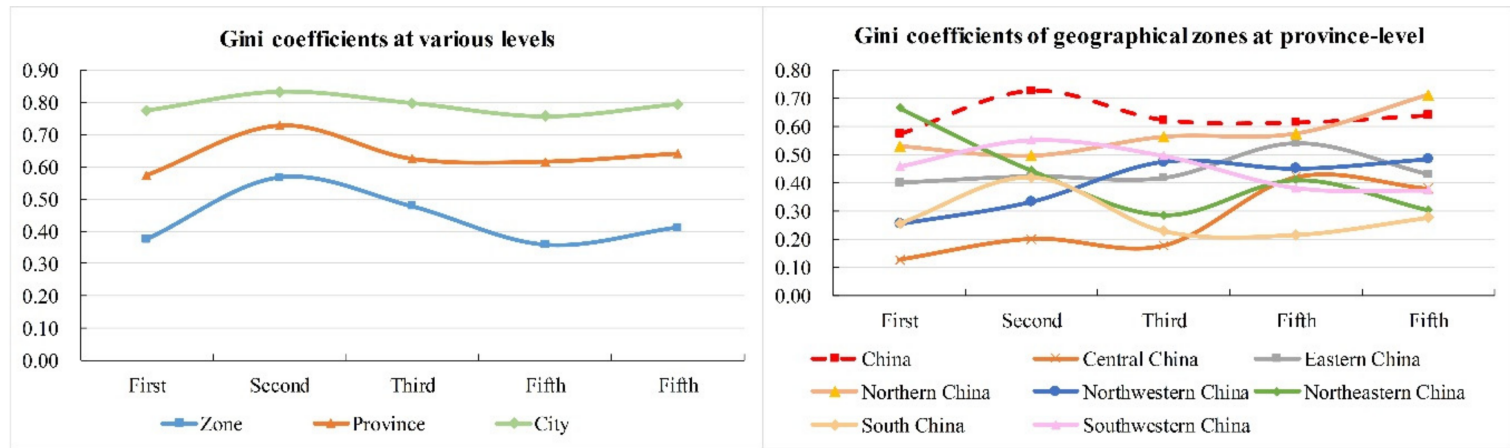

Figure 3. The value of Gini coefficients (a) at various levels and (b) in seven geographic zones.

In other words, finding the spatial heterogeneity of the distribution of traditional villages is easier with a smaller spatial scale. Specifically, the results above illustrate that traditional villages show the strongest polarization characteristics at the municipal level, which is always higher than 0.75 and once reached above 0.8 . The Gini coefficients at the provincial level show medium disparity in the distribution of traditional villages. Compared with the municipal and provincial levels, the coefficients at the zone level have decreased significantly, indicating that traditional villages are relatively evenly distributed at the zone level. The variation trend and spatial pattern of the distribution differences of traditional villages on a single scale are one-sided. Relevant studies must pay more attention to the scale sensitivity and scale dependence of traditional village distributions, which can reveal that the smaller the spatial scale is, the greater the difference in the distribution of villages is and the more significant the spatial agglomeration characteristics of traditional village spatial patterns are. The results above show that the protection of traditional villages of different scales should be targeted through precise measures, and the local measures should be closely based on the spatiotemporal differentiation process and state of traditional villages of different scales.

For each geographic zone (Figure 3b), the Gini coefficients of northern China are largest and show a trend of increasing with each batch, illustrating that traditional villages in northern China are the least clustered and that the distribution is the most uneven due to the many divided villages in northern China. The Gini coefficients of eastern China fluctuate between 0.40 and 0.59 , illustrating that the distributions of traditional villages are not equal. The change trends of the southwestern and southern 
regions are consistent with those of the whole country. In comparison, the distributions of southern China are much more concentrated and uniform. Both the trend and level of southwestern China are almost consistent with those of the whole country. The reason is that traditional villages in the southwest are the most concentrated and densely distributed in China. The distribution characteristics of the southwest can reflect the striking features and characteristics of China. Central China shows obvious changes from a concentrated distribution to an uneven distribution. The polarized distribution existed in the early period in northeast China but gradually changed to relatively balanced development. The main reason may also be that each batch of traditional villages in the northeast contains very few villages. The Gini coefficient in the north-western region has increased significantly, illustrating that the distribution of traditional villages is unbalanced.

\subsection{Hot Spot Analysis Representing Clustering Patterns}

Figures 4 and 5 illustrate the hot spot analysis results of the spatial distribution and temporal variations, respectively, of traditional villages at the province and city scales. Overall, the spatial patterns of traditional Chinese villages show an aggregated trend with local fluctuations. The first, second and fifth clusters tend to be strengthened, while the third and fourth clusters tend to be weakened. The occurrence probability of hot spots and cold spots approved by traditional villages has significant local characteristics. At the provincial scale, the distribution pattern differed across the study period: the number of high-density areas in the southwest decreased, and the provinces located in eastern China showed an increased number of traditional villages. The overall trend of hot spot distribution is in eastern and south-eastern China, illustrating that traditional villages are clustered in these areas. Only Hunan and Guangxi provinces are persistent hot spots in the five batches of traditional villages. Yunnan, Chongqing and Guizhou are oscillating hot spots. The cold spots in the provinces of north-eastern China are shrinking. The cold spots were concentrated in north-eastern and northern China during the study period, especially in Jilin Province. In addition, the distribution of the first batch is most similar to the distribution of all batches.

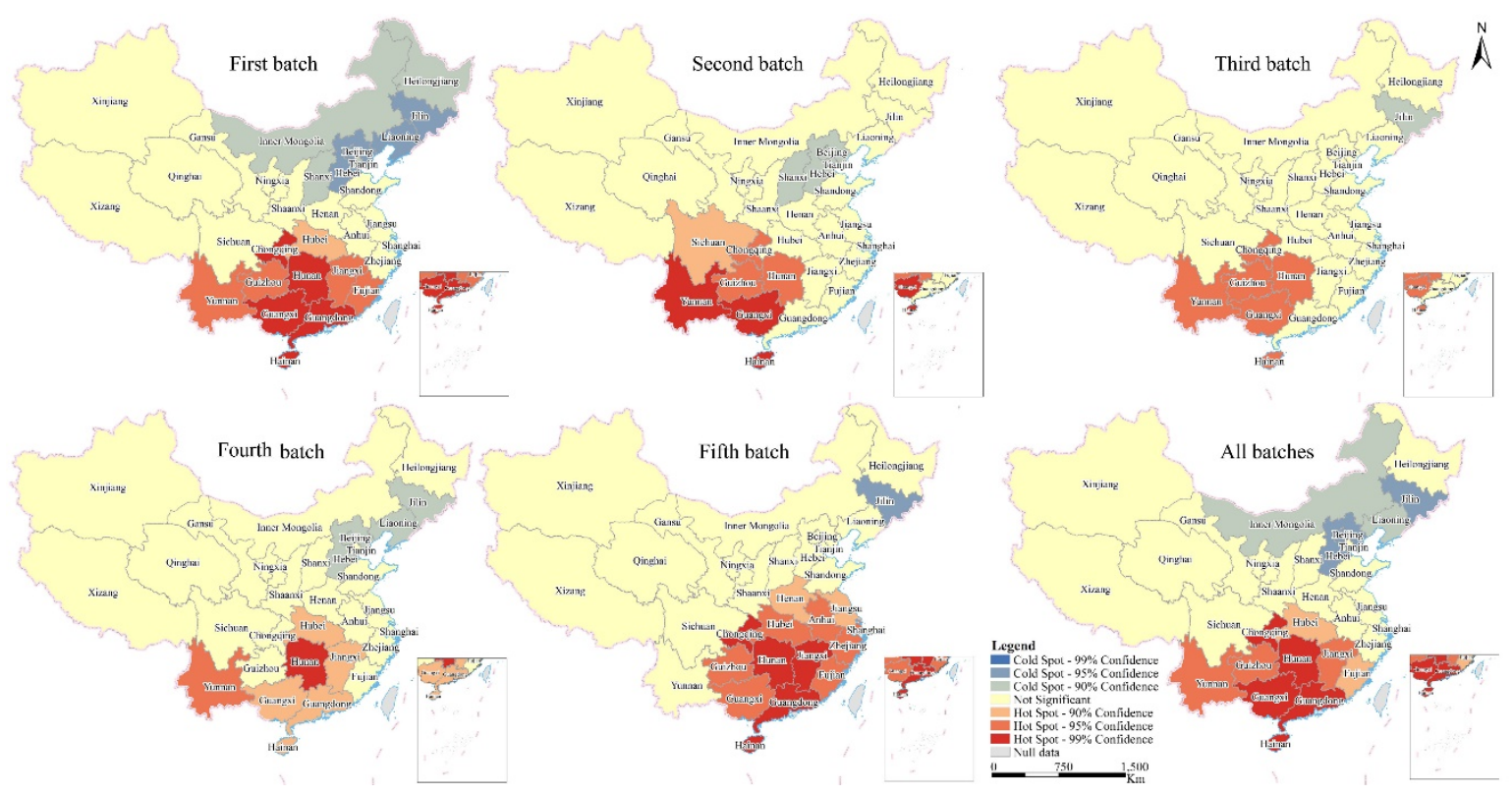

Figure 4. Spatiotemporal hot spot analysis at the provincial scale. 


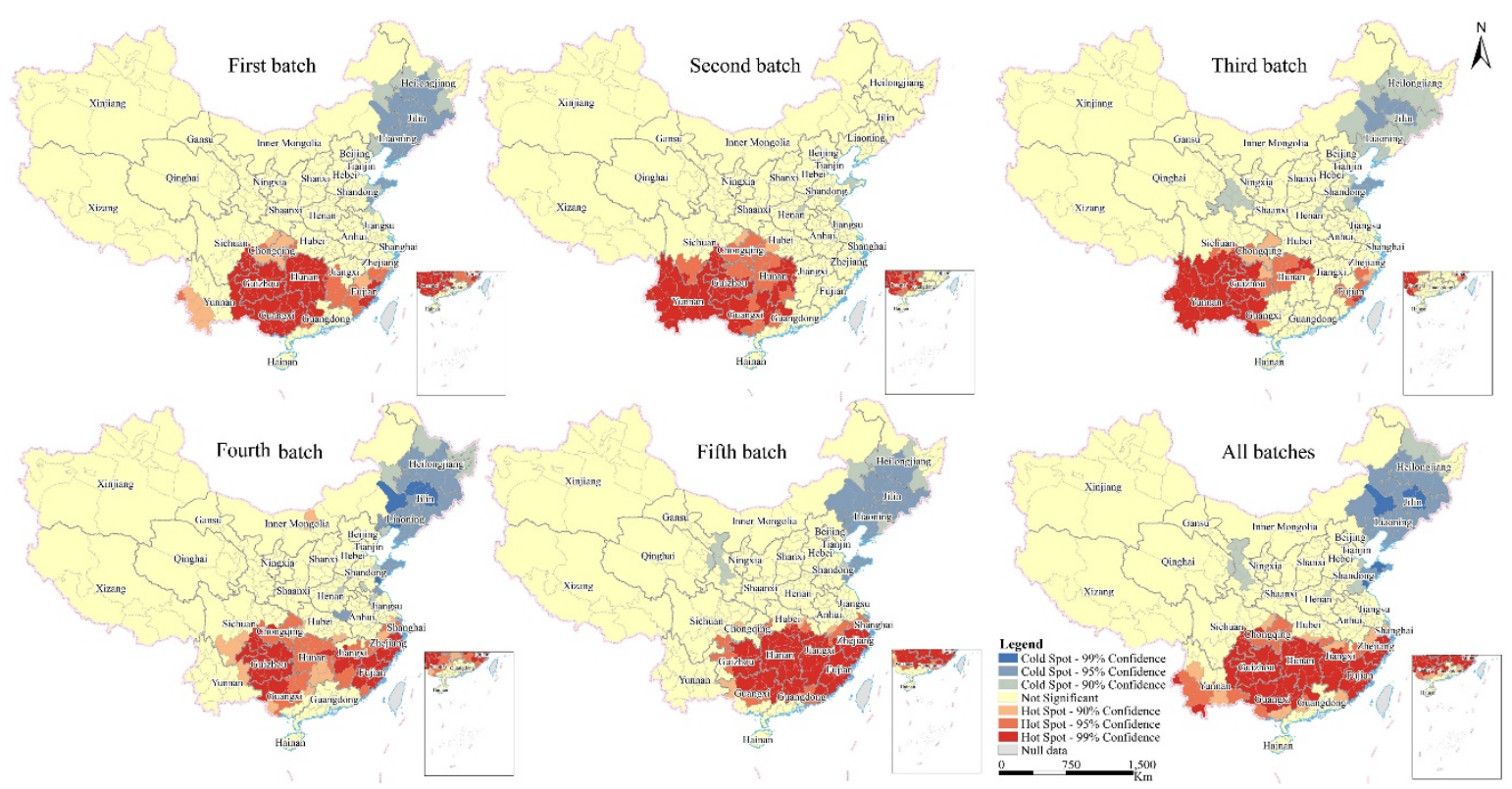

Figure 5. Spatiotemporal hot spot analysis at the city scale.

Compared with the hot spot analysis results at the province scale, the results at the city scale can reflect more detailed potential and some different information. The most notable characteristics are as follows: (1) some cities in Gansu, Shandong, Henan Jiangsu and Qinghai are cold spots. The cities in Inner Mongolia are no longer cold spots. Instead, the fourth batch of traditional villages has a hot spot in Inner Mongolia. (2) The provinces including Sichuan, Hunan, Guizhou, Chongqing and Yunnan all have cities that are persistent hot spots. (3) The spatial agglomeration patterns of traditional villages at the city scale are relatively scattered and specific in local areas. Figures 4 and 5 both reveal that traditional villages in China mainly have the characteristics of hot spots in terms of spatial distribution. The hot spots are concentrated in the southwest and southeast while the cold spots are concentrated in the northeast, showing a significant north-south difference.

Combining the results of the hot spot analysis, the spatiotemporal distribution of Chinese villages can be characterized by "more in the south and less in the north and more clustered in the south and more dispersed in the north", and they are influenced by natural conditions and human factors. Specifically, the natural conditions including landform, climate, soil, vegetation and hydrology have an important influence on the distribution of villages. Human factors including traffic location, population density and economic development also have significant effects on the distribution and development of villages. For example, the Sichuan Basin has unique topographic conditions, a warm and humid climate and fertile and high-yield soil, which can provide a good living environment for the formation and aggregation of villages. The traditional villages are clustered in the areas that are moist and warm.

\subsection{WSDE and the Trajectories of WSDE Centers}

\subsubsection{All of China}

The geometrical trajectories of the WSDE indicate different spatial patterns and temporal changing trends of traditional village distributions at each scale of the analysis unit. Figure 6 shows the WSDEs and the trajectories of the WSDE centers in China. The centers of the WSDE have always deviated to the southeast of China's geographic center. The locations of the WSDE centers are indicated by the temporal and spatial variations in movements from the east-west-east, showing that the approval of traditional villages began to shift to the east. Zhejiang, Fujian, Anhui and Hunan have further increased financial support, technological investment and special advice for the protection of traditional villages to improve the rural environment of traditional villages. These results are consistent with the results of 
the hot spot analysis in Figures 4 and 5. The oblateness first increased from 0.119 to 0.382 and then decreased to 0.083 , demonstrating that the orientation of the traditional village distribution is becoming less obvious throughout China. In general, from the perspective of the whole country, a difference exists between the long and the short half axes of the ellipse, indicating that the distribution of traditional villages has a certain direction, and the expression direction of the long half axes is from northeast to southwest. Although an angle exists between the boundary line of $\mathrm{Hu}$ Huanyong, the direction is the same, indicating that population distribution is an important factor affecting the distribution of traditional villages. According to the changes in the long and short half axes, the distribution direction and scope have significantly weakened. The detailed results of the temporal WSDEs can be seen in Table S1 in the Supporting Information file. The WSDEs of all of China reflect only macro trends, and the microscopic details are analyzed by specific geographic zones and the containing provinces as follows.

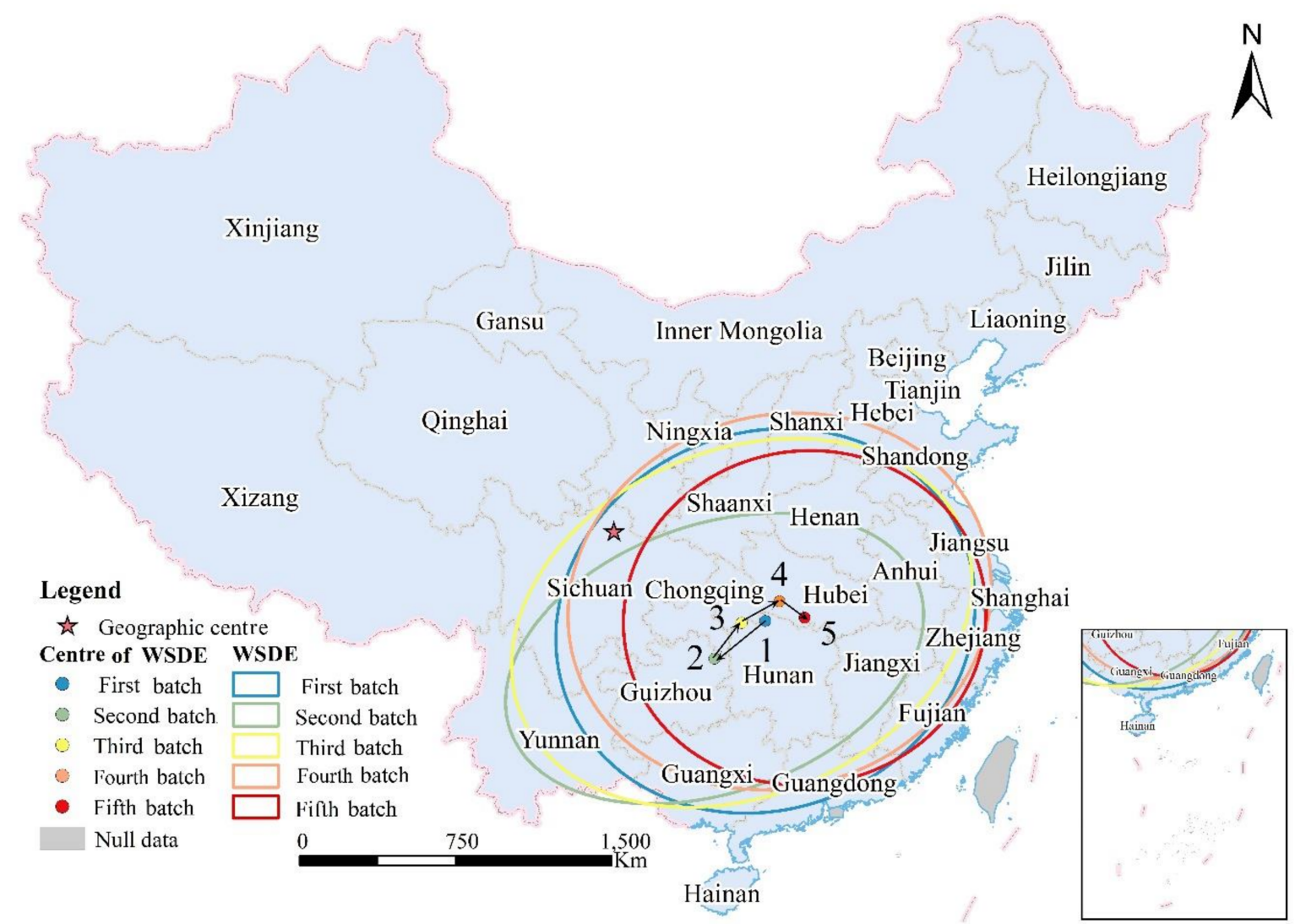

Figure 6. Weighted standard deviation ellipses (WSDEs) and trajectories of WSDE centers in China.

\subsubsection{The Geographic Zones}

The WSDEs and the trajectories of WSDE centers for seven geographic zones and subordinate provinces are listed in the following figures. The detailed results of the temporal WSDEs can be seen in Table S2 in the Supporting Information file. For Central China (Figure 7), the WSDE centers of the first three batches are all around the geographic centers of Central China, illustrating that the distributions of the first three batches of traditional villages are relatively uniform. The fourth and fifth batches of traditional villages deviate to the southwest of the geographic center of Central China (i.e., Hunan Province). This result is inseparable from Hunan Province's increasing financial input, rational use of resources, innovation of protection and development mode (i.e., classification protection), and promotion of ecological agriculture development, primary processing of agricultural products and other characteristic industries. The deviation illustrates that the recent traditional villages of Central 
China are concentrated in Hunan Province. In terms of stability, the moving trajectory of the WSDE centers is relatively large, indicating that the stability of traditional village aggregation centers is weak and that the degree of village clustering is low. This region is characterized by great topographical fluctuations. In low-altitude plain areas such as Jianghan Plain, Dongting Lake Plain and Poyang Lake Plain, due to high degree of urbanization, the villages are not easily preserved; therefore, few traditional villages exist at present. Nevertheless, due to the relatively harsh natural environment, remote location, traffic congestion, and slow economic development in the areas with high altitude, the traditional villages are easy to preserve.

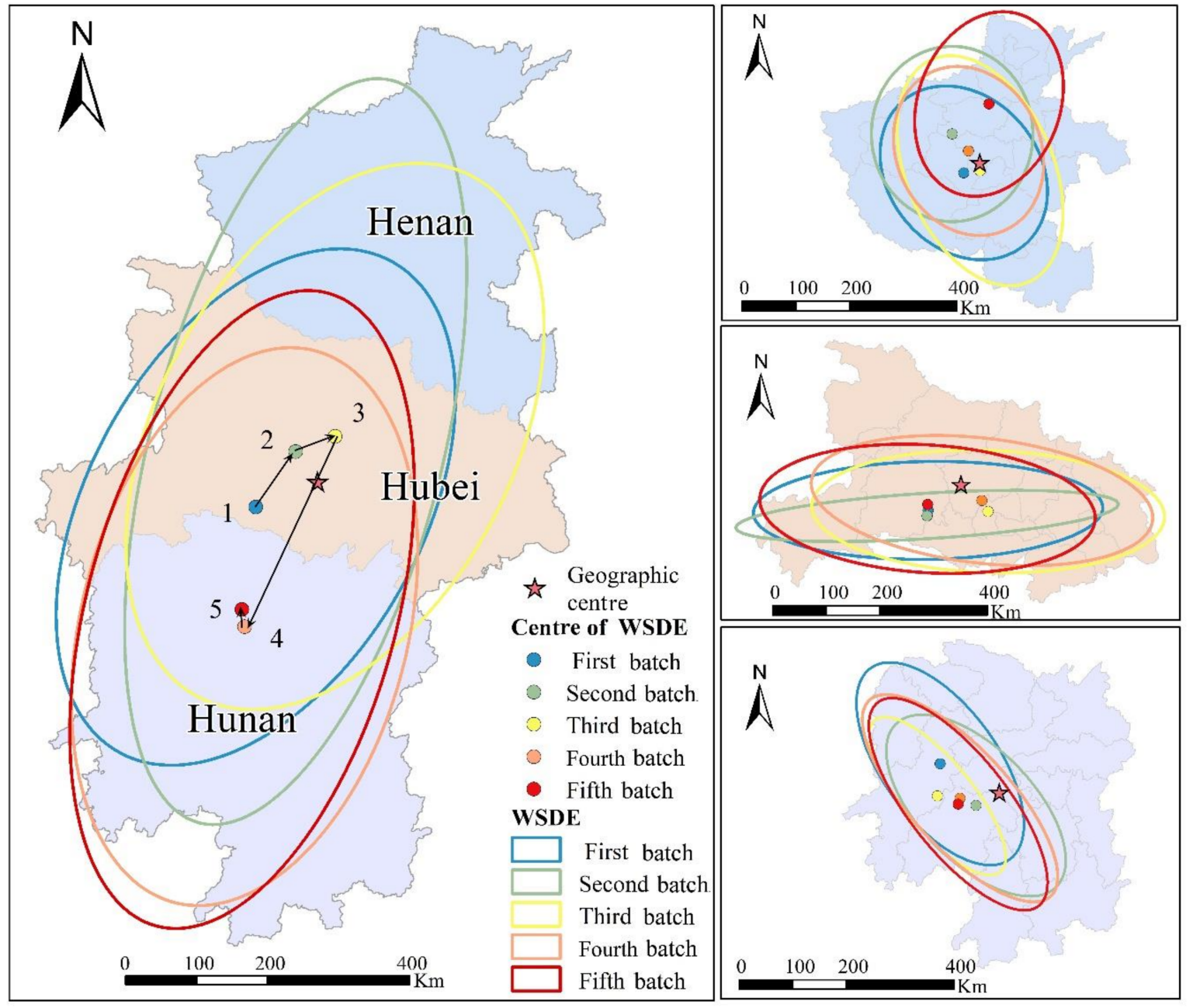

Figure 7. The WSDEs and the trajectories of centers for Central China and subordinate provinces.

For eastern China (Figure 8), the ranges of WSDE center movement trajectories are small, the stability of traditional village aggregation centers is good, and the degree of aggregation of villages is high. In addition, the trajectories of the centers are annular, which means that the distribution pattern of traditional villages associated with the WSDE is highly clustered within eastern China, and the aggregation effect in eastern China presents a small deviation from the whole. All WSDE centers are located to the southeast of the geographic center of eastern China, demonstrating that the spatial distribution of traditional villages has obvious heterogeneity. The villages are more heavily concentrated in Zhejiang, Jiangxi and Fujian. The shapes of the WSDEs also reflect that the distributions of each batch of traditional villages have little difference in time, i.e., the WSDEs show temporal stability. The eastern coastal areas have unique geographic advantages including favorable climatic conditions, fertile soil, and relatively rich water resources. More importantly, the rapid development of the regional economy and the reasonable distribution of transportation infrastructure greatly promoted the development of 
non-agricultural industries in the region. Therefore, the clustering distribution characteristics of villages are obvious.
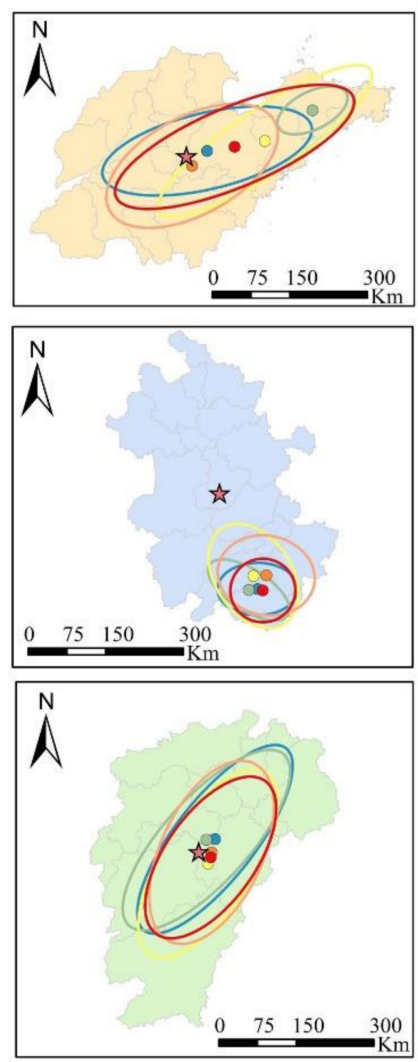
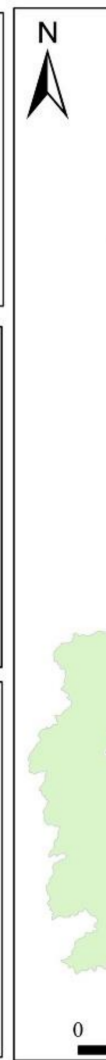
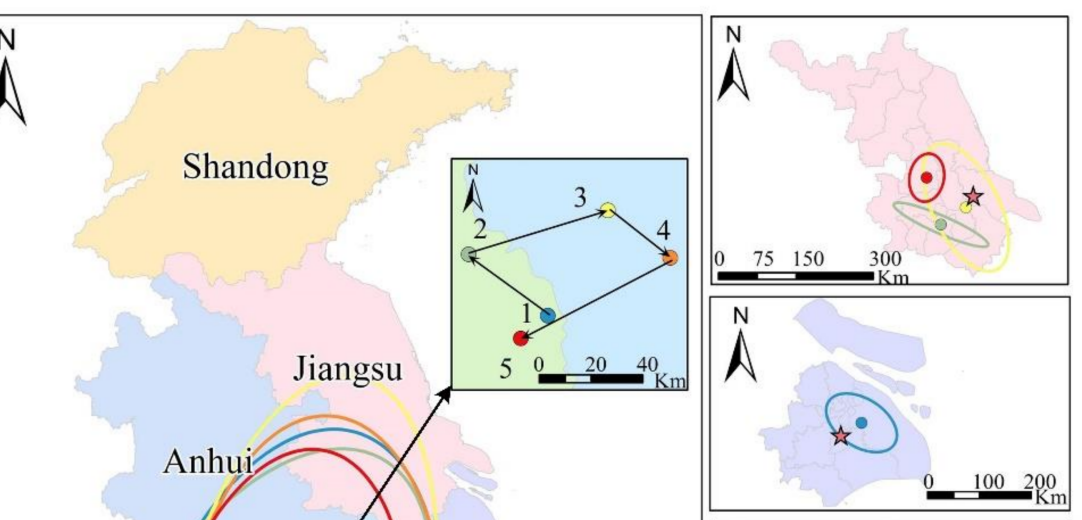

Shandong

Figure 8. The WSDE and the center trajectories for eastern China and subordinate provinces.

The spatiotemporal patterns of southern China (Figure 9) are similar to the patterns of eastern China. The oblateness of southern China is smaller than that of eastern China, illustrating that the distribution direction of traditional villages in southern China is not as strong as that in eastern China. The range of the center movement trajectory is small and shows a ring structure, indicating that the traditional villages in southern China are relatively stable in time and space, with little difference in aggregation. Notably, the fourth batch of traditional villages shows an obvious change in direction and center location, which shows a north-south direction and transforms to Guangxi Province. In comparison, the second batch of traditional villages has a more obvious distribution direction and discrete degree.

Southwestern China is a famous zone with a large number of traditional villages (Figure 10). In terms of WSDE shapes, the WSDEs of the first three groups of traditional villages are flatter than those of the last two groups, indicating that the orientation of the traditional village distribution in southwestern China has weakened. The increase in the semi-minor axis shows a greater dispersion of traditional villages in southwestern China, with a weaker centripetal force. The locations of WSDE centers are near the geographic center of southwestern China, and they are concentrated in Yunnan and Guizhou. The trajectories of the centers are obviously circular, and the range of movement trajectory is small, illustrating that the stability of the traditional village aggregation center in southwestern China is good, and the aggregation effect presents a small deviation. The southern region is mountainous and hilly, with greater topographic relief. Obvious differences exist in regional resources, environmental conditions and minority culture in these areas, and the traditional villages tend to show large-scale clustering in areas with relatively superior environmental conditions. 


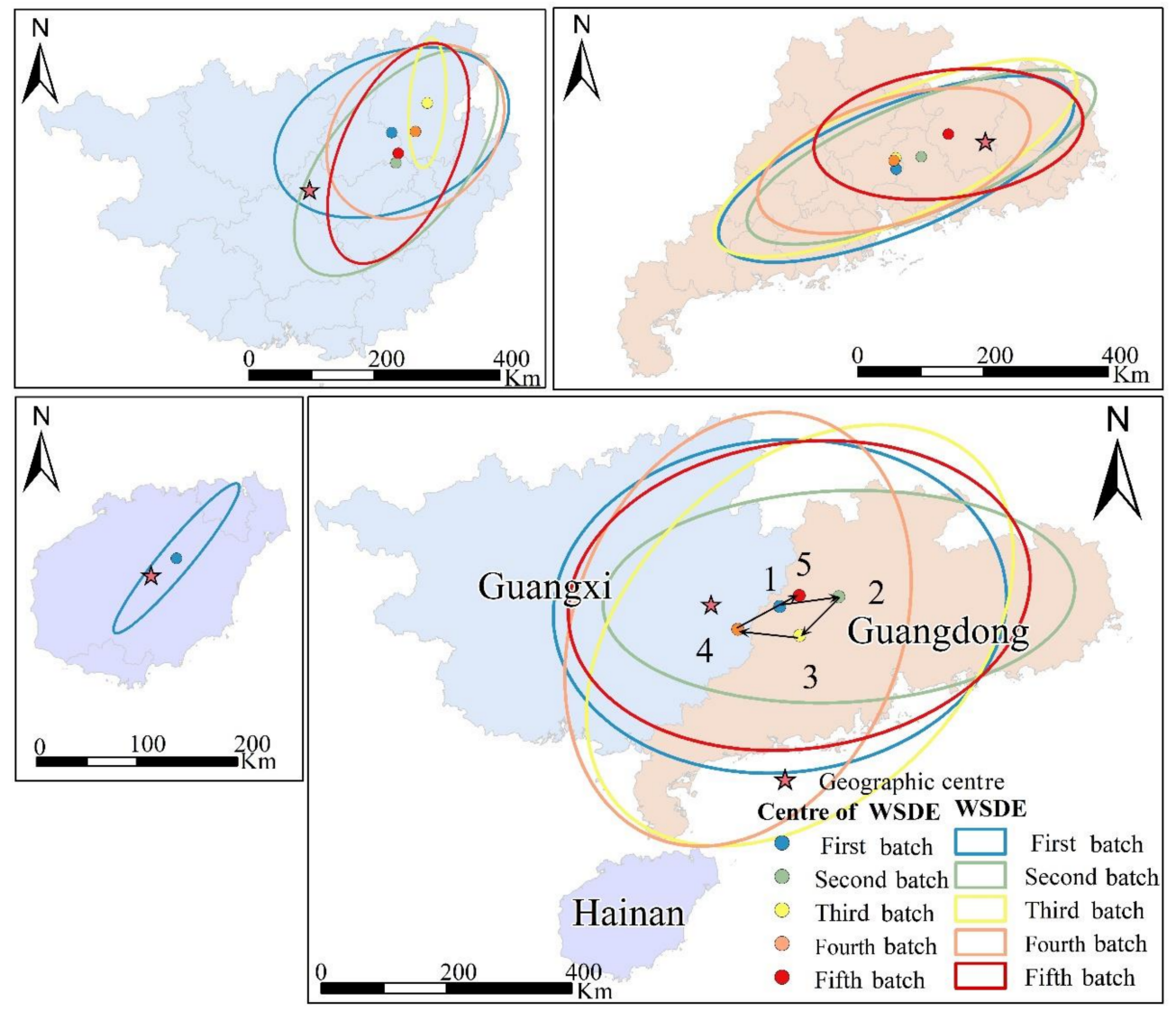

Figure 9. The WSDE and the center trajectories for southern China and subordinate provinces.

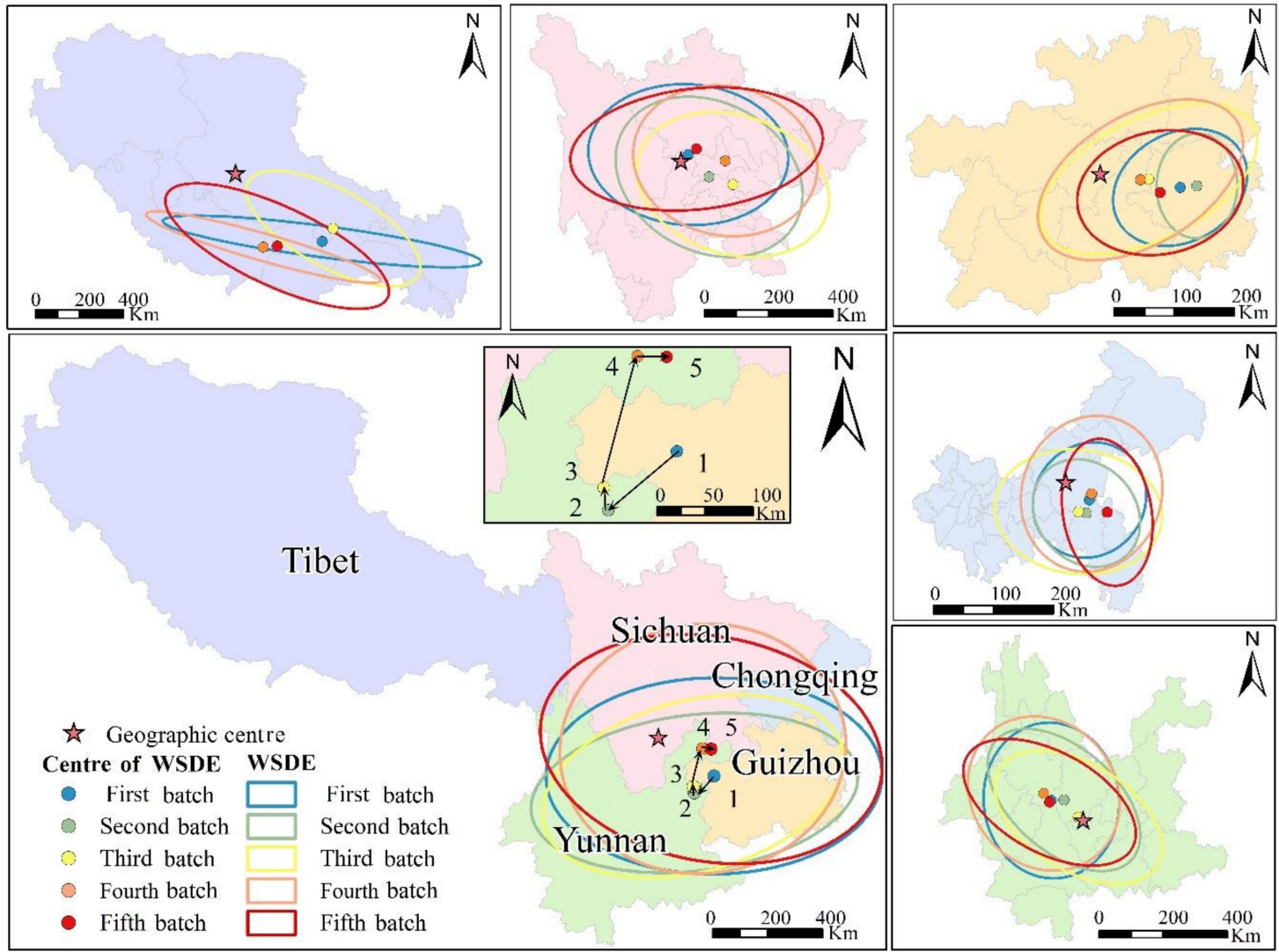

Figure 10. The WSDEs and the trajectories of centers for southwestern China and subordinate provinces. 
Compared with other zones, the spatiotemporal patterns of the traditional villages in northern China have unique characteristics (Figure 11). Except for the second batch of traditional villages, the traditional village distribution directions of all other batches are obvious (i.e., southwest-northeast). The short radius of the WSDEs is much smaller than the long radius, and the distribution of each batch of traditional villages has a strong centripetal force. The movement range of the WSDE centers is small, and the stability of the aggregated centers of traditional villages is good, namely, concentrated in Shanxi Province. However, the movement trajectory of the WSDE center is characterized by vibration, indicating that the distribution of traditional villages associated with the WSDE has periodicity and timeliness within the research scope.

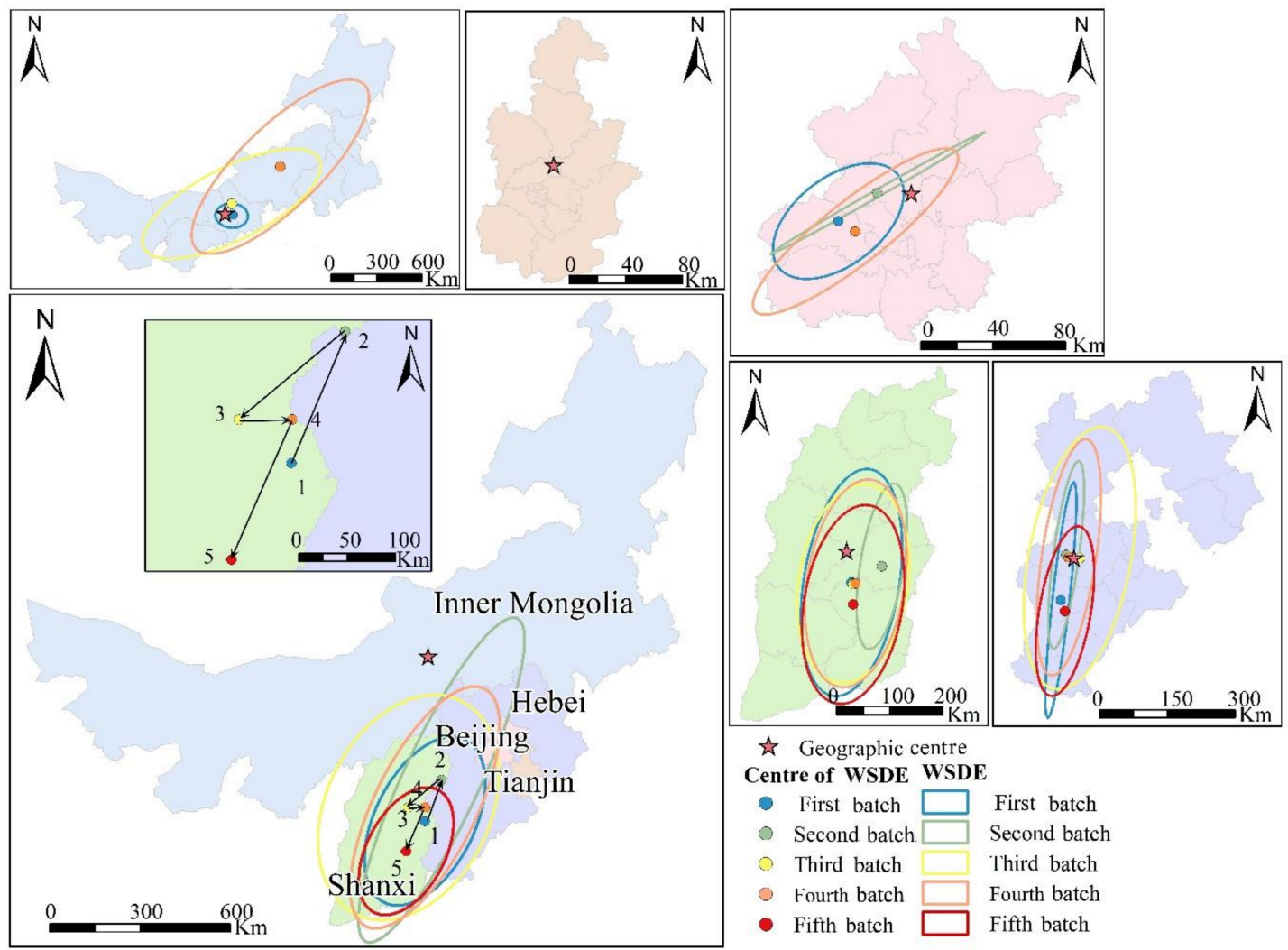

Figure 11. The WSDEs and center trajectories for northern China and subordinate provinces.

The number of traditional villages in north-eastern China is currently the lowest in China (Figure 12). The shapes of the WSDEs and the trajectories of the WSDE centers are slightly disorganized. First, because the number of traditional villages is small, the first batch of traditional villages cannot be used to construct WSDEs due to insufficient vertices. Second, the directions and shapes of WSDEs vary greatly among the different batches, showing that the distribution randomness of traditional villages in north-eastern China is strong, and the stability of aggregation centers is weak over time. The spatial distribution of villages shows dispersive characteristics with a small scale resulting from the traditional small-scale peasant economy development model in northeast China.

For north-western China (Figure 13), the WSDEs mainly cover Qinghai, Gansu, Ningxia and Shaanxi Provinces, whereas Xinjiang is barely covered, illustrating that few traditional villages exist in Xinjiang. Based on the shapes of the WSDEs, the distribution directions and degrees of aggregation of traditional villages have changed. The first three batches of traditional villages are relatively dispersed, showing a south-northwest direction. The distribution of the last two groups of traditional villages is more concentrated, showing an east-west direction. According to the movement scope and trajectories of WSDE centers, the first three groups of traditional villages have a small difference in distribution and are relatively stable while the last two groups of traditional villages show few differences. 


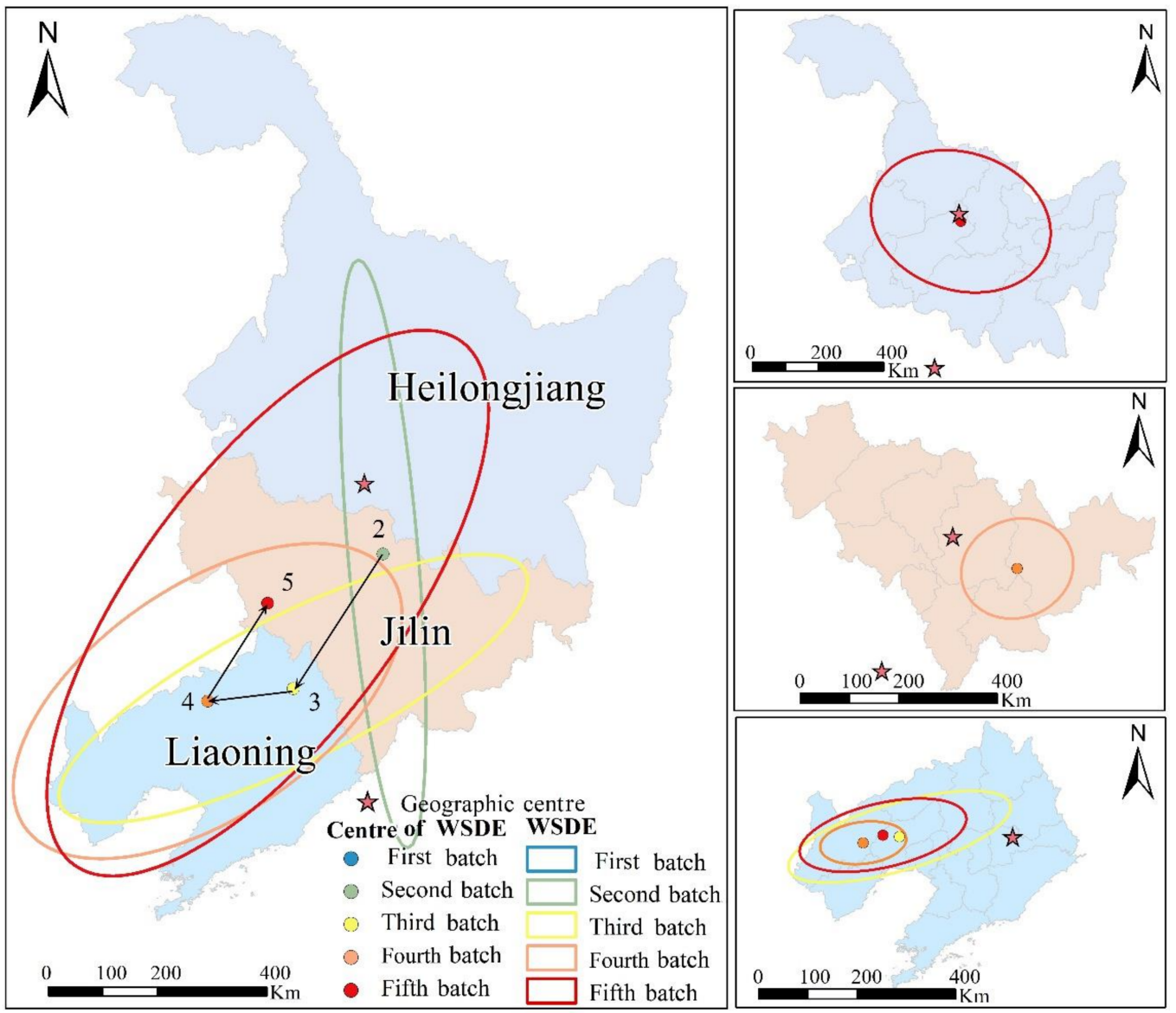

Figure 12. The WSDEs and the center trajectories for north-eastern China and subordinate provinces.

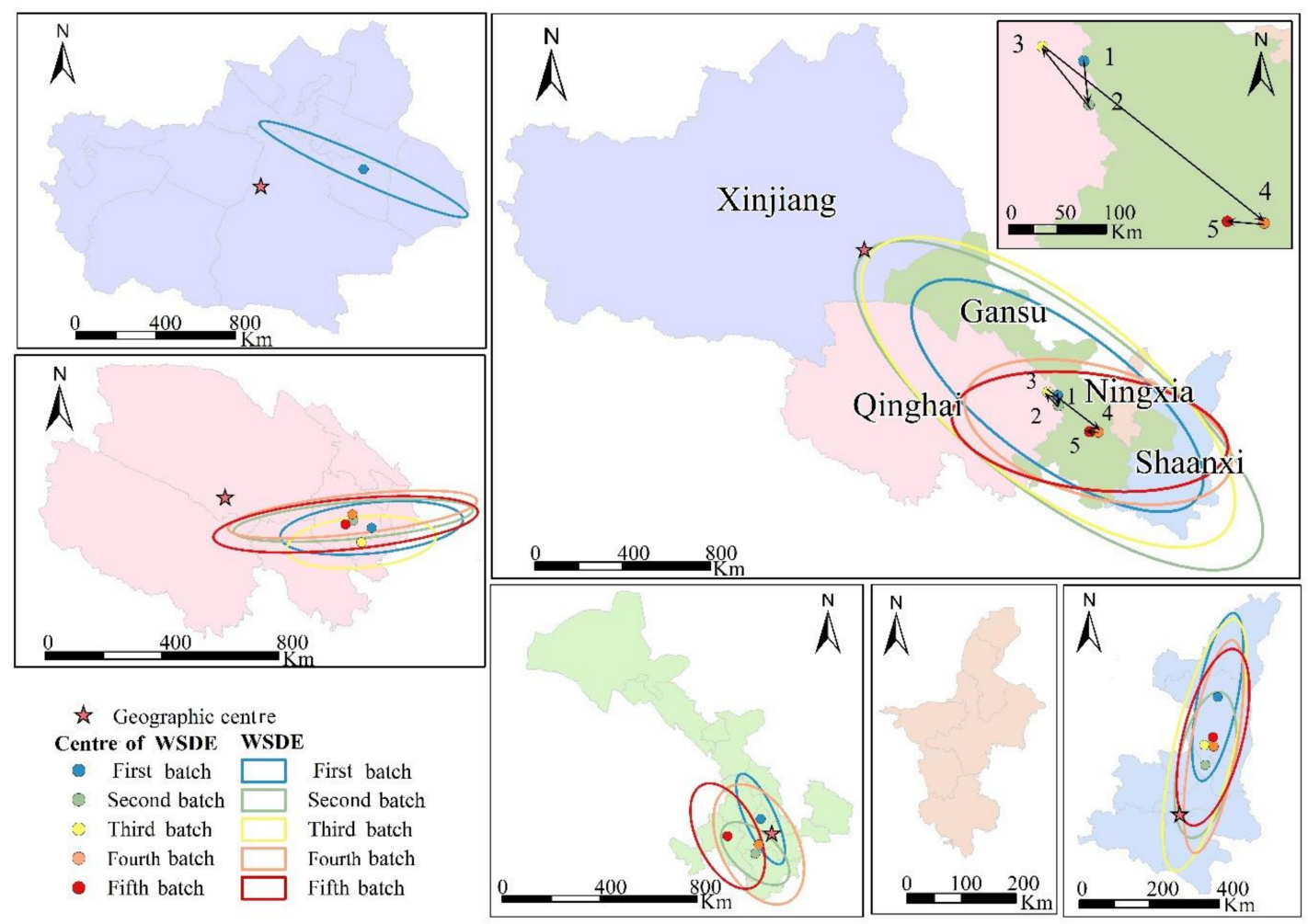

Figure 13. The WSDE and the center trajectories for north-western China and subordinate provinces. 


\subsubsection{The Provincial Scale}

The shapes of the center trajectories of the WSDEs indicate that the traditional villages in each province present different spatial and temporal patterns (Figures 7-13, Tables S3-S9). In general, by comparing the length and short axis, oblateness, center location and movement trajectory of each province's WSDE, the following conclusions can be obtained:

(1) The index of oblateness can be used to determine the direction and degree of centripetal force. In total, it can be roughly divided into the following types: (1) inconspicuous directivity: Henan, Zhejiang, Sichuan, Heilongjiang, and Jilin; (2) obvious directivity: Hubei, Hunan, Henan, Jiangxi, Shandong, Guangdong, Hebei, Shanxi, Liaoning, Gansu, Qinghai, Shanxi, and Xinjiang; and (3) alternate directions: Anhui, Fujian, Guangxi, Chongqing, Guizhou, Guangxi, Yunan, Beijing, and Inner Mongolia.

(2) The locations of the WSDE centers relative to the geographic center of the province can reflect the administrative patterns and temporal stability of traditional village distributions. The groups are as follows: (1) near the geographic center with stability: Jiangxi, Zhejiang, and Fujian; (2) near the geographic center with vibration: Henan, Hubei, Inner Mongolia, Hebei, Gansu, and Sichuan; (3) off the geographic center with stability: Hubei, Anhui, Liaoning, Qinghai, Guangxi, Chongqing, and Yunnan; (4) off the geographic center with vibration: Jiangsu, Shandong, Tianjin, Shanxi, Shaanxi, Guangdong, Tibet, and Guizhou; and (5) other patterns: Shanghai, Tianjin, Jilin, Heilongjiang, Xinjiang, Ningxia, and Hainan.

\section{Discussion and Conclusions}

Due to the imbalance of economic status, cultural deposits, natural conditions, population distribution, etc., the distributions of traditional villages have geographic differences. Moreover, different geographic spatial scales have different spatial differentiation characteristics, and different times (i.e., different batches) also have different spatial differentiation changes in traditional villages. The spatial pattern of five batches of traditional villages can reflect the interdependence of rural geographic elements and the stage of socioeconomic transformation and development, which are of great significance to the regional promotion and implementation of a rural revitalization strategy. Moreover, considering the five batches of traditional villages with uniform criteria, the evolution of approved traditional villages can analyze the change process and development of traditional villages in different scales and reflect the changes and differences of relevant influencing factors, such as economic input, policy support and rural vitality, etc. Therefore, this article discusses the spatiotemporal distribution characteristics of villages based on the proposed " $3 \mathrm{M}$ " framework. The spatial and temporal processes of traditional village development can reflect the spatiotemporal characteristics of urban development, especially the patterns of regional development and urbanization. The measurement of multidimensional indicators for identifying spatial and temporal distribution of traditional villages can help policy makers to formulate specific and targeted policies or projects for traditional villages and rural development so as to improve the "shortest board" of rural economic development and environmentally sustainable development and to seek breakthroughs in promoting effective and sustainable development of rural economic reconstruction and urban-rural integration. This article has positive significance for the study and analysis of traditional villages, and it has certain reference value for the protection, development and utilization decisions of regional ancient villages. The policy implications based on the corresponding results can be summarized as follows:

(1) From the spatial dimension, the distribution of traditional villages is very uneven, and it is concentrated in southern and eastern China. This uneven geographic distribution is consistent with the distribution of the "Hu Huanyong Line", which divides China into roughly equal but very different parts [63]. The coverage area and the centers of the WSDEs are mainly to the southeast of the "Hu Huanyong Line", illustrating that the regional differences in natural factors (such as terrain, slope direction, and land use) and anthropogenic factors (such as population 
pattern, regional rural development level, financial assistance, cultural corridor and historical and cultural background) make traditional village distributions different $[64,65]$. From temporal dimension, it is apparent that the number of identified traditional villages is increasing year by year. In order to protect traditional villages effectively, the types of protection of traditional villages are being further improved, the intensity of protection are being strengthened and the scope of protection is being expanded during identification. What is more, the public awareness of protecting traditional villages is increasing. For western China, the most fundamental thing is to promote the development of local economy and society, construct villager ideology, increase financial input from the governments, encourage the participation and support of social forces and support characteristic industries, which can provide opportunities for young people to work, and prevent the "empty-nest" situation and "aging" of traditional villages in this region. Therefore, the traditional villages can be preserved and developed better. For eastern and southern China, which have clusters of traditional villages, the primary task should be to further stimulate the vitality, capacity, motivation and competitiveness of traditional village development. The development of traditional villages in the east and south is relatively new; thus, protecting the buildings and structures of villages as well as preventing overdevelopment and protecting the traditional culture contained in them is necessary. The dynamic inheritance of traditional villages should give play to their own advantages, seize the opportunity for rural tourism, and combine the development of traditional villages with ecological agriculture, characteristic folk custom service, agricultural landscape and promotion of characteristic agricultural products [7]. In brief, the construction of traditional villages should be placed in the regional environment and in future development, and integrated, long-term and localized planning should be made for village architecture, industrial layout, history and culture.

(2) The results of the various spatial analysis methods in this paper show that the spatial distribution of traditional villages differs with location, shape and density at different scales. That is, the study of distribution differences of traditional villages is highly sensitive to changes in spatial scale. In terms of the analysis of cold hot spots, the comparison between the provincial scale and municipal scale shows that the smaller the spatial scale is, the greater the difference and agglomeration of traditional villages are; meanwhile, the smaller the spatial scale is, the easier it is to find the spatial heterogeneity of traditional village distribution. The analysis based on the results of multiple scales is not only conducive to accurately grasping the distribution law of traditional villages but also critical to the precision of the exploration and analysis of the factors influencing traditional cultural villages. Based on a comprehensive analysis of the multiscale spatiotemporal characteristics of traditional villages in China, this article summarizes and proposes the basic principles and countermeasures for the coordinated development of traditional villages. 1) Focusing on the coordinated development of traditional villages on a small scale and classifying traditional villages with common features to protect them from multilevel perspectives is important [66]. For example, the special opinion of Zhejiang that divided traditional villages into "ancient architectural villages", "natural ecological villages", "folk villages" and other types can be implemented in other provinces. 2) Paying attention to the development of the whole system of traditional village groups in the region is wise [67]. According to the main regional characteristics of traditional villages, the government can innovate and build industry and product systems related to the development and revitalization of traditional villages in the region. For example, in the eastern region, traditional village tourism routes with stories of "Huizhou merchants in southern Anhui", "the land of fish and rice in Jiangsu and Zhejiang", and "the red base areas in Jiangxi" can be designed to not only spread the diverse culture of traditional villages but also improve the development of industries and economics. 3) Although the spatiotemporal distribution of traditional villages shows obvious differences at diverse scales, the advances in new computer technology, network communication technology and virtual simulation technology, the proposed national strategic system and improvement 
of the internationalization level provide equal opportunities for the local development of rural areas [68]. For example, the city of Huangshan in Anhui Province has applied virtual simulation technology to achieve digital protection of traditional villages.

This article explores the spatiotemporal distribution characteristics of traditional villages, which are of certain reference value to decision-making related to the protection, development and utilization of Chinese villages. However, this study still leaves room for future explorations. Studies on the differences in Chinese traditional villages are highly sensitive to spatial scales. The construction and optimization of a multi-scale and multi-mechanism composite theoretical framework is necessary for future studies on the distribution of traditional villages. First, the precision of traditional village analyses can be more refined, such as at the county scale [69]. Relevant studies can further support the studies on regional imbalances in China. Second, the factors influencing the survival of traditional villages, including the natural environment, social economy, regional policies and historical culture, remain to be studied. Moreover, this article only selects some factors for qualitative evaluation and explanation. Further research is needed to quantitatively analyze the factors affecting the distribution differences of traditional villages. The factors influencing the distribution differences of traditional villages at different scales still need to be determined to promote the protection and development of rural areas. In combination with future relevant data collection, we will explore the spatiotemporal effects of determinants on traditional villages at different spatial scales.

Supplementary Materials: The following are available online at http://www.mdpi.com/2073-445X/9/11/449/s1, Table S1: Changes of related parameters of traditional villages' WSDEs in China. Table S2. The related parameters of traditional villages' WSDEs in geographical zones. Table S3. The related parameters of traditional villages' WSDEs in provinces of central China. Table S4. The related parameters of traditional villages' WSDEs in provinces of eastern China. Table S5. The related parameters of traditional villages' WSDEs in provinces of southern China. Table S6. The related parameters of traditional villages' WSDEs in provinces of southwestern China. Table S7. The related parameters of traditional villages' WSDEs in provinces of northern China. Table S8. The related parameters of traditional villages' WSDEs in provinces of north-eastern China. Table S9. The related parameters of traditional villages' WSDEs in provinces of north-western China

Author Contributions: Data curation, C.W. and M.C.; Funding acquisition, C.W., W.W. and L.Z.; Investigation, C.W. and M.C.; Methodology, W.W. and C.W.; Project administration, C.W.; Software, C.W. and X.L.; Visualization, C.W. and M.C.; Writing-original draft, C.W. and W.W.; Writing—review and editing, X.L., W.W., and L.Z. All authors have read and agreed to the published version of the manuscript.

Funding: This research was funded by the National Natural Science Foundation of China (No. 41901326, 42001237, 42071212); the Natural Science Foundation of Jiangsu Province (BK20190742); the Philosophy and Social Science Research of Jiangsu Higher Education Institutions (2019SJA0097) and the Open Research Fund Program of the Key Laboratory of Digital Mapping and Land Information Application Engineering (NASGZRZYBWD201906).

Conflicts of Interest: The authors declare no conflict of interest.

\section{References}

1. Hu, X.; Li, H.; Zhang, X.; Chen, X.; Yuan, Y. Multi-dimensionality and the totality of rural spatial restructuring from the perspective of the rural space system: A case study of traditional villages in the ancient Huizhou region, China. Habitat Int. 2019, 94, 102062. [CrossRef]

2. Wang, H.; Chiou, S. Study on the Sustainable Development of Human Settlement Space Environment in Traditional Villages. Sustainability 2019, 11, 4186. [CrossRef]

3. Dumreicher, H. Chinese villages and their sustainable future: The European Union-China-Research Project "SUCCESS". J. Environ. Manag. 2008, 87, 204-215. [CrossRef] [PubMed]

4. Tan, Y.; He, J.; Han, H.; Zhang, W. Evaluating residents' satisfaction with market-oriented urban village transformation: A case study of Yangji Village in Guangzhou, China. Cities 2019, 95, 102394. [CrossRef]

5. Session, U.C.G. Recommendation on the Safeguarding of Traditional Culture and Folklore: Adopted by the General Conference at Its Twenty-fifth Session, Paris, 15 November 1989; Unesco: Paris, France, 1989.

6. Guo, Z.; Sun, L. The planning, development and management of tourism: The case of Dangjia, an ancient village in China. Tour. Manag. 2016, 56, 52-62. [CrossRef] 
7. Li, Y.; Fan, P.; Liu, Y. What makes better village development in traditional agricultural areas of China? Evidence from long-term observation of typical villages. Habitat Int. 2019, 83, 111-124. [CrossRef]

8. Yang, R.; Xu, Q.; Long, H. Spatial distribution characteristics and optimized reconstruction analysis of China's rural settlements during the process of rapid urbanization. J. Rural. Stud. 2016, 47, 413-424. [CrossRef]

9. Govindan, K.; Loisi, R.V.; Roma, R. Greenways for rural sustainable development: An integration between geographic information systems and group analytic hierarchy process. Land Use Policy 2016, 50, 429-440.

10. Su, S.; Zhang, Q.; Zhang, Z.; Zhi, J.; Wu, J. Rural settlement expansion and paddy soil loss across an ex-urbanizing watershed in eastern coastal China during market transition. Reg. Environ. Chang. 2011, 11, 651-662. [CrossRef]

11. Long, H.; Tu, S.; Ge, D.; Li, T.; Liu, Y. The allocation and management of critical resources in rural China under restructuring: Problems and prospects. J. Rural Stud. 2016, 47, 392-412. [CrossRef]

12. Fink, M.; Lang, R.; Harms, R. Local responses to global technological change-Contrasting restructuring practices in two rural communities in Austria. Technol. Forecast. Soc. Chang. 2013, 80, 243-252. [CrossRef]

13. Guo, Y.; Xiao, Y.; Yuan, Q. The redevelopment of peri-urban villages in the context of path-dependent land institution change and its impact on Chinese inclusive urbanization: The case of Nanhai, China. Cities 2017, 60, 466-475. [CrossRef]

14. Tan, M.; Li, X. The changing settlements in rural areas under urban pressure in China: Patterns, driving forces and policy implications. Landsc. Urban Plan. 2013, 120, 170-177. [CrossRef]

15. Zhu, J.; Zhu, M.; Xiao, Y. Urbanization for rural development: Spatial paradigm shifts toward inclusive urban-rural integrated development in China. J. Rural Stud. 2019, 71, 94-103. [CrossRef]

16. Lu, Y.; Song, S.; Wang, R.; Liu, Z.; Meng, J.; Sweetman, A.J.; Jenkins, A.; Ferrier, R.C.; Li, H.; Luo, W. Impacts of soil and water pollution on food safety and health risks in China. Environ. Int. 2015, 77, 5-15. [CrossRef] [PubMed]

17. Gao, J.; Wu, B. Revitalizing traditional villages through rural tourism: A case study of Yuanjia Village, Shaanxi Province, China. Tour. Manag. 2017, 63, 223-233. [CrossRef]

18. Kamada, M.; Nakagoshi, N. Influence of cultural factors on landscapes of mountainous farm villages in western Japan. Landsc. Urban Plan. 1997, 37, 85-90. [CrossRef]

19. Choi, Y.K. The spatial structure of power: Traditional villages and houses in Korea. Environ. Plan. B Plan. Des. 2003, 30, 589-603. [CrossRef]

20. Crouch, D. Popular culture and what we make of the rural, with a case study of village allotments. J. Rural Stud. 1992, 8, 229-240. [CrossRef]

21. Chen, B.; Nakama, Y. A study on village forest landscape in small island topography in Okinawa, Japan. Urban For. Urban Green. 2010, 9, 139-148. [CrossRef]

22. Saleh, M.A.E. The decline vs the rise of architectural and urban forms in the vernacular villages of southwest Saudi Arabia. Build. Environ. 2001, 36, 89-107. [CrossRef]

23. Kastenholz, E.; Carneiro, M.J.; Marques, C.P.; Lima, J. Understanding and managing the rural tourism experience-The case of a historical village in Portugal. Tour. Manag. Perspect. 2012, 4, 207-214. [CrossRef]

24. Lepp, A. Residents' attitudes towards tourism in Bigodi village, Uganda. Tour. Manag. 2007, $28,876-885$. [CrossRef]

25. Zhang, R.; Xi, J.; Wang, S.; Wang, X.; Ge, Q. Village network centrality in rural tourism destination: A case from Yesanpo tourism area, China. J. Mt. Sci. 2015, 12, 759-768. [CrossRef]

26. Yao, G.; Xie, H. Rural spatial restructuring in ecologically fragile mountainous areas of southern China: A case study of Changgang Town, Jiangxi Province. J. Rural Stud. 2016, 47, 435-448. [CrossRef]

27. Zhou, G.; He, Y.; Tang, C.; Yu, T.; Xiao, G.; Zhong, T. Dynamic mechanism and present situation of rural settlement evolution in China. J. Geogr. Sci. 2013, 23, 513-524. [CrossRef]

28. Chen, H.; Wang, X. Exploring the relationship between rural village characteristics and Chinese return migrants' participation in farming: Path dependence in rural employment. Cities 2019, 88, 136-143. [CrossRef]

29. Hu, D.; Reardon, T.; Rozelle, S.; Timmer, P.; Wang, H. The emergence of supermarkets with Chinese characteristics: Challenges and opportunities for China's agricultural development. Dev. Policy Rev. 2004, 22, 557-586. [CrossRef]

30. Long, H.; Liu, Y.; Li, X.; Chen, Y. Building new countryside in China: A geographical perspective. Land Use Policy 2010, 27, 457-470. [CrossRef] 
31. Liu, Y.; Li, J.; Yang, Y. Strategic adjustment of land use policy under the economic transformation. Land Use Policy 2018, 74, 5-14. [CrossRef]

32. McDonagh, J. Rural geography I: Changing expectations and contradictions in the rural. Prog. Hum. Geogr. 2013, 37, 712-720. [CrossRef]

33. Gilg, A.W. An introduction to rural geography. Trans. Inst. Br. Geogr. 1985, 11, 374.

34. Yang, R.; Liu, Y.; Long, H.; Qiao, L. Spatio-temporal characteristics of rural settlements and land use in the Bohai Rim of China. J. Geogr. Sci. 2015, 25, 559-572. [CrossRef]

35. Bohua, L.; Sha, Y.; Peilin, L.; Yindi, D. Spatial Distribution of Traditional Villages and Influence Factors in Hunan Province. J. Landsc. Res. 2016, 8, 63-67.

36. Wu, C.; Ren, F.; Hu, W.; Du, Q. Multiscale geographically and temporally weighted regression: Exploring the spatiotemporal determinants of housing prices. Int. J. Geogr. Inf. Sci. 2019, 33, 489-511. [CrossRef]

37. Liu, D.J.; Hu, J.; Chen, J.; XV, X. The study of spatial distribution pattern of traditional villages in China. China Popul. Resour. Environ. 2014, 24, 157-162.

38. Yang, X.; Fang, C.; Zhu, K. A study of spatial-temporal pattern and influencing factors of traditional villages in Shaanxi Province. In Proceedings of IOP Conference Series: Earth and Environmental Science; IOP Publishing: Bristol, UK, 2019; p. 052011.

39. Overman, H.G. Can we learn anything from economic geography proper? J. Econ. Geogr. 2004, 4, 501-516. [CrossRef]

40. Wu, J.; Jelinski, D.E.; Luck, M.; Tueller, P.T. Multiscale analysis of landscape heterogeneity: Scale variance and pattern metrics. Geogr. Inf. Sci. 2000, 6, 6-19. [CrossRef]

41. Wu, C.; Ren, F.; Ye, X.; Liang, X.; Du, Q. Spatiotemporal analysis of multiscale income mobility in China. Appl. Geogr. 2019, 111, 102060. [CrossRef]

42. Wu, Y.; Sun, X.; Sun, L.; Choguill, C.L. Optimizing the governance model of urban villages based on integration of inclusiveness and urban service boundary (USB): A Chinese case study. Cities 2020, 96, 102427. [CrossRef]

43. Su, S.; Xiao, R.; Zhang, Y. Multi-scale analysis of spatially varying relationships between agricultural landscape patterns and urbanization using geographically weighted regression. Appl. Geogr. 2012, 32, 360-375. [CrossRef]

44. Liao, F.H.; Wei, Y.D. Space, scale, and regional inequality in provincial China: A spatial filtering approach. Appl. Geogr. 2015, 61, 94-104. [CrossRef]

45. Esfehani, M.H.; Albrecht, J.N. Roles of intangible cultural heritage in tourism in natural protected areas. J. Herit. Tour. 2018, 13, 15-29. [CrossRef]

46. Sayre, N.F. Ecological and geographical scale: Parallels and potential for integration. Prog. Hum. Geogr. 2005, 29, 276-290. [CrossRef]

47. Goodchild, M.F. Scale in GIS: An overview. Geomorphology 2011, 130, 5-9. [CrossRef]

48. Kang, J.Y.; Zhang, J.H.; Hu, H.; Zhou, J.; Xiong, J.; Jingyao, K. Analysis on the spatial distribution characteristics of Chinese traditional villages. Prog. Geogr. 2016, 35, 839-850.

49. Zhu, J. Transition of villages during urbanization as collective communities: A case study of Kunshan, China. Cities 2018, 72, 320-328. [CrossRef]

50. Qu, Y.; Jiang, G.; Zhao, Q.; Ma, W.; Zhang, R.; Yang, Y. Geographic identification, spatial differentiation, and formation mechanism of multifunction of rural settlements: A case study of 804 typical villages in Shandong Province, China. J. Clean. Prod. 2017, 166, 1202-1215. [CrossRef]

51. Yang, D.; Luan, W.; Qiao, L.; Pratama, M. Modeling and spatio-temporal analysis of city-level carbon emissions based on nighttime light satellite imagery. Appl. Energy 2020, 268, 114696. [CrossRef]

52. Dong, L.; Liang, H. Spatial analysis on China's regional air pollutants and $\mathrm{CO}_{2}$ emissions: Emission pattern and regional disparity. Atmos. Environ. 2014, 92, 280-291. [CrossRef]

53. Li, Y.; Wei, Y.D. The spatial-temporal hierarchy of regional inequality of China. Appl. Geogr. 2010, 30, 303-316. [CrossRef]

54. Arbia, G. The role of spatial effects in the empirical analysis of regional concentration. J. Geogr. Syst. 2001, 3, 271-281. [CrossRef]

55. Shalit, H.; Yitzhaki, S. Mean-Gini, portfolio theory, and the pricing of risky assets. J. Financ. 1984, 39, 1449-1468. [CrossRef] 
56. Ord, J.K.; Getis, A. Local spatial autocorrelation statistics: Distributional issues and an application. Geogr. Anal. 1995, 27, 286-306. [CrossRef]

57. Zheng, B.; Myint, S.W.; Fan, C. Spatial configuration of anthropogenic land cover impacts on urban warming. Landsc. Urban Plan. 2014, 130, 104-111. [CrossRef]

58. Kaba, E.; Philpot, W.; Steenhuis, T. Evaluating suitability of MODIS-Terra images for reproducing historic sediment concentrations in water bodies: Lake Tana, Ethiopia. Int. J. Appl. Earth Obs. Geoinf. 2014, 26, 286-297. [CrossRef]

59. Mitchel, A. The ESRI Guide to GIS analysis, Volume 2: Spartial measurements and statistics. In The ESRI Guide to GIS Analysis; Esri Press: Redlands, CA, USA, 2005.

60. Villagra, P.; Rojas, C.; Ohno, R.; Xue, M.; Gómez, K. A GIS-base exploration of the relationships between open space systems and urban form for the adaptive capacity of cities after an earthquake: The cases of two Chilean cities. Appl. Geogr. 2014, 48, 64-78. [CrossRef]

61. Ayhan, I.; Cubukcu, K.M. Explaining historical urban development using the locations of mosques: A GIS/spatial statistics-based approach. Appl. Geogr. 2010, 30, 229-238. [CrossRef]

62. Li, L.; Yang, L.; Zhu, H.; Dai, R. Explorative analysis of Wuhan intra-urban human mobility using social media check-in data. PLOS ONE 2015, 10, e0135286. [CrossRef]

63. $\mathrm{Hu}, \mathrm{H}$. Distribution of China's population. Acta Geogr. Sin. 1935, 2, 33-74.

64. Ma, L.; Chen, M.; Fang, F.; Che, X. Research on the spatiotemporal variation of rural-urban transformation and its driving mechanisms in underdeveloped regions: Gansu Province in western China as an example. Sustain. Cities Soc. 2019, 50, 101675. [CrossRef]

65. Zhou, J.; He, P.; Qin, Y.; Ren, D. A selection model based on SWOT analysis for determining a suitable strategy of prefabrication implementation in rural areas. Sustain. Cities Soc. 2019, 50, 101715. [CrossRef]

66. Wu, C.; Peng, N.; Ma, X.; Li, S.; Rao, J. Assessing multiscale visual appearance characteristics of neighbourhoods using geographically weighted principal component analysis in Shenzhen, China. Comput. Environ. Urban Syst. 2020, 84, 101547. [CrossRef]

67. Lin, Y.; De Meulder, B.; Wang, S. Understanding the 'village in the city'in Guangzhou: Economic integration and development issue and their implications for the urban migrant. Urban Stud. 2011, 48, 3583-3598. [CrossRef]

68. Astuti, K.A.; Darma, G.S. Community-based tourism: Measuring readiness of artificial intelligence on traditional village. Int. J. Soc. Sci. Humanit. 2019, 3, 81-89. [CrossRef]

69. He, S.; Liao, F.H.; Li, G. A spatiotemporal analysis of county economy and the multi-mechanism process of regional inequality in rural China. Appl. Geogr. 2019, 111, 102073. [CrossRef]

Publisher's Note: MDPI stays neutral with regard to jurisdictional claims in published maps and institutional affiliations.

(C) 2020 by the authors. Licensee MDPI, Basel, Switzerland. This article is an open access article distributed under the terms and conditions of the Creative Commons Attribution (CC BY) license (http://creativecommons.org/licenses/by/4.0/). 